\title{
Massive Non-Encephalitic Neurocysticercosis
}

\author{
José L. Ruiz-Sandoval ${ }^{1,2}$, Guadalupe Ramírez-Guzmán ${ }^{3}$, \\ Erwin Chiquete $^{4}$ and Ángel Vargas-Sánchez ${ }^{1}$
}

Key words: brain, cestoda, cysticercosis, epilepsy, Mexico, seizures

(Intern Med 52: 1435, 2013)

(DOI: 10.2169/internalmedicine.52.0315)

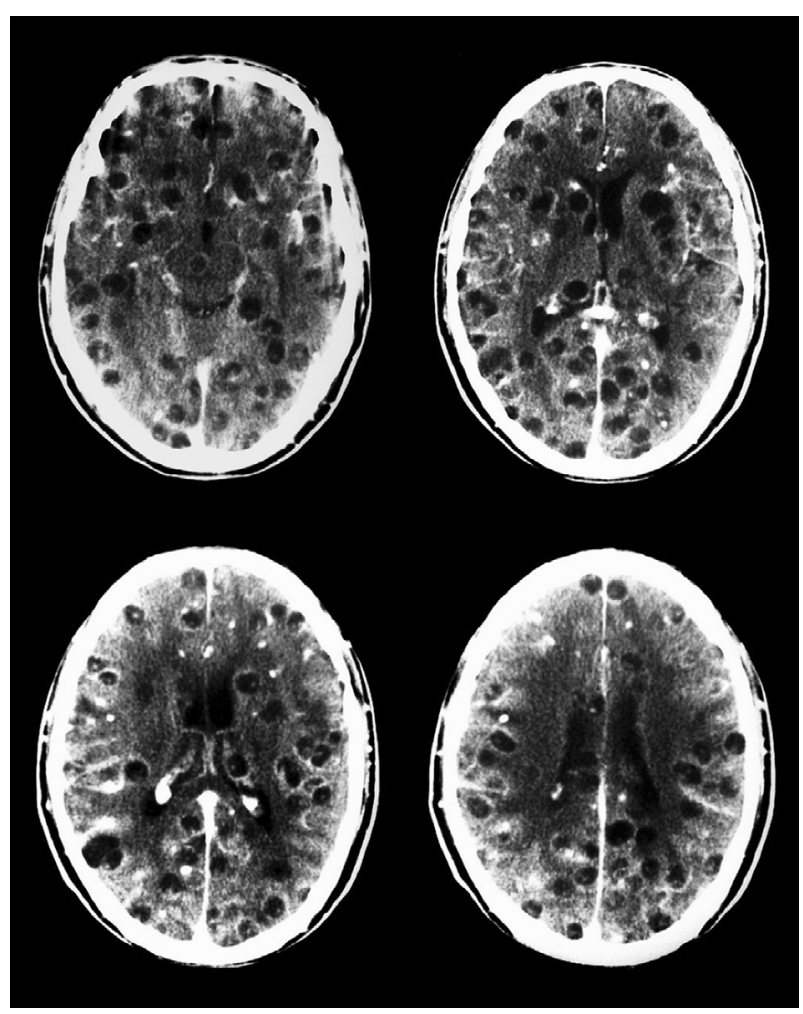

Picture.

A 45-year-old garbage collector was referred to our department with a history of tonic-clonic seizures and risky sexual behavior (anilingus). A neurological examination was normal. Contrast-enhanced cranial CT showed calcified lesions and viable parasites compatible with a diagnosis of massive non-encephalitic neurocysticercosis. Oral metallic implants impeded performing brain MRI. Hepatitis and HIV serologies were negative. The patient was discharged with steroids and an anticonvulsant. Delayed cysticidal therapy was planned; however, albendazole therapy was immediately initiated in another hospital, which led to brain edema, uncontrolled seizures, rostrocaudal deterioration and death.

Cestoda infections are rare in developed countries (1). In contrast, neurocysticercosis is a leading cause of adult-onset epilepsy in Latin America. Massive infections are classified as encephalitic or non-encephalitic (2). In patients with the encephalitic presentation, cysticidal drugs can cause extensive parasite lysis and aggravate brain inflammation (2). In patients with non-encephalitic massive neurocysticercosis, cysticidal therapy is usually considered; (2) however, rapid initiation of antiparasitic medications can launch an encephalitic process.

The authors state that they have no Conflict of Interest (COI).

\section{References}

1. Nakamura-Uchiyama F, Kobayashi K, Ohnishi K. An imported case of disseminated cysticercosis and taeniasis. Intern Med 51: 347-348, 2012.

2. Del Brutto OH, Campos X. Massive neurocysticercosis: encephalitic versus non-encephalitic. Am J Trop Med Hyg 87: 381, 2012.

\footnotetext{
${ }^{1}$ Department of Neurology, Civil Hospital of Guadalajara, Mexico, ${ }^{2}$ Department of Neurosciences, University Center of Health Sciences, University of Guadalajara, Mexico, ${ }^{3}$ Department of Neuroradiology, Civil Hospital of Guadalajara, Mexico and ${ }^{4}$ Department of Neurology and Psychiatry, National Institute of Medical Sciences and Nutrition, Mexico

Received for publication February 11, 2013; Accepted for publication February 25, 2013

Correspondence to Dr. José L. Ruiz-Sandoval, jorulej-1nj@prodigy.net.mx

(C) 2013 The Japanese Society of Internal Medicine Journal Website: http://www.naika.or.jp/imonline/index.html
} 